\title{
Laser based fabrication of chitosan mediated silver nanoparticles.
}

\begin{abstract}
We report fabrication of silver nanoparticles (Ag NPs) by laser ablation technique in different concentrations of aqueous chitosan solution. The ablation process of silver plate was carried out by using a nanosecond Q-switched Nd:YAG pulsed laser and the characterization of Ag NPs was done by Transmission electron microscopy, UV-Vis spectroscopy, and X-ray diffraction. UV-visible plasmon absorption spectra revealed that the formation efficiency as well as the stability of nanoparticles was increased by addition of chitosan. On the other hand, the size decrement of nanoparticles was more remarkable in the higher chitosan concentration.
\end{abstract}

Keyword: Laser Ablation; Silver nanoparticles; Chitosan. 